\title{
Linx
}

Revue des linguistes de l'université Paris X Nanterre

$41 \mid 1999$

L'hypothétique

\section{Le champ des subordonnées dites conditionnelles du français : conditions, éventualités, suppositions et hypothèses}

Sarah de Vogüé

\section{OpenEdition}

Journals

Édition électronique

URL : http://journals.openedition.org/linx/1251

DOI : 10.4000/linx.1251

ISSN : 2118-9692

Éditeur

Presses universitaires de Paris Nanterre

Édition imprimée

Date de publication : 1 décembre 1999

Pagination : $93-118$

ISBN : 0246-8743

ISSN : 0246-8743

Référence électronique

Sarah de Vogüé, «Le champ des subordonnées dites conditionnelles du français : conditions,

éventualités, suppositions et hypothèses », Linx [En ligne], 41 | 1999, mis en ligne le 27 août 2012

consulté le 19 avril 2019. URL : http://journals.openedition.org/linx/1251 ; DOI : 10.4000/linx.1251

Ce document a été généré automatiquement le 19 avril 2019

Département de Sciences du langage, Université Paris Ouest 


\title{
Le champ des subordonnées dites conditionnelles du français : conditions, éventualités, suppositions et hypothèses
}

\author{
Sarah de Vogüé
}

1 Le champ des subordonnées dites conditionnelles du français est quantitativement important, puisque l'on peut dénombrer au moins trente marqueurs (pour l'essentiel des locutions conjonctives, mais aussi un certain nombre de constructions parataxiques du type Il ouvre la bouche, j'hurle) susceptibles d'introduire une telle subordonnée. Sur le plan des valeurs, on constate au contraire une sorte d'homogénéité qui est tout aussi remarquable ${ }^{1}$ : l'essentiel du champ est constitué de constructions qui, pour diverses qu'elles soient, n'en sont pas moins quasi synonymes. Ainsi, par delà des différences plus ou moins perceptibles, les quelques exemples réunis ci-dessous en (1) paraissent, du point de vue du moins de l'expression de la condition, être globalement équivalents :

(1) Si on l'invite, il est content.

On l'invite, il est content.

L'invite-t-on, il est content.

Au cas où on l'invite, il est content.

Dans l'hypothèse où on l'invite, il est content.

A supposer qu'on l'invite, il est content.

Pour peu qu'on l'invite, il est content.

On l'invite, il est content.

Pourvu qu'on l'invite, il est content.

Moyennant qu'on l'invite, il est content.

Du moment qu'on l'invite, il est content.

A condition qu'on l'invite, il est content.

On ne peut appréhender la configuration de la classe de marqueurs concernée sans prendre au sérieux cette relation de quasi-synonymie : à la fois il y a des différences, à la fois ces différences peuvent être neutralisées. 
3 Cette situation brouillée doit être mise en relation avec le rôle particulier que joue la conjonction si dans la constitution de la classe ${ }^{2}$. Si est généralement considéré comme le marqueur prototypique de la condition. De fait, il fonctionne comme substitut universel pour tous les marqueurs de la classe : en l'occurrence, c'est même cette substitution qui tout à la fois garantit l'appartenance à la classe de tel marqueur (on soutiendra que l'on a affaire à un énoncé conditionnel dès lors que le marqueur qui y apparaît peut être remplacé par si), et fonde la relation de quasi-synonymie décrite ci-dessus (d'une certaine façon, les marqueurs paraissent quasi-synonymes dans la mesure où ils peuvent tous être remplacés par si). Cela ne veut pas dire pour autant que si garde toujours la même valeur d'une substitution à l'autre. On sait que si est polysémique, et il l'est même jusqu'à pouvoir prendre des valeurs qui ne relèvent plus du champ de la condition :

(2) S'il est riche, c'est parce qu'il a gagné au loto.

(3) S'il est riche, il n'est pas milliardaire.

(4) S'il est riche, cela ne se voit pas.

4 (2) est un exemple de si explicatif : l'apodose fournit une explication à p, pris pour acquis, et ce faisant vise en quelque sorte à le banaliser (il n'y a rien d'anormal à ce qu'il soit riche, il ne l'est que parce que q). (3) est un exemple de si concessif : p est alors admis (concédé, comme dans tout énoncé concessif), et q sert à le minimiser. (4) enfin est un exemple d'un si que j'ai proposé d'appeler dialectique dans de précédents travaux ${ }^{3}$ : loin d'être concédé, $\mathrm{p}$ est donné comme vraisemblablement faux, q figurant alors un argument pour le disqualifier. Ces trois valeurs ne peuvent pas être tenues pour des valeurs conditionnelles ${ }^{4}$.

Le caractère paradoxal d'une telle situation ne doit pas être minimisé : il se trouve que le marqueur prototypique de la condition est aussi celui qui, à force d'exploiter toutes les potentialités du champ conditionnel, va jusqu'à remettre en cause la clôture de ce champ ${ }^{5}$

Tous ces phénomènes nous conduisent à envisager la notion de condition comme étant une notion foncièrement élastique : elle l'est au sens où elle est compatible avec toutes les différenciations que parcourt si et qu'expriment les autres marqueurs; et elle l'est au point de permettre des déformations telles qu'elles en viennent à ne plus relever de la condition.

7 C'est bien une élasticité de cet ordre qui se manifeste dans les descriptions traditionnelles. Ainsi constate-t-on par exemple que la catégorie est parfois rassemblée au titre de la condition, parfois au titre de l'hypothèse. De plus, la description associe généralement les deux notions, soit de manière explicite, en les mettant en relation, soit en passant d'un terme à l'autre, au gré des besoins, ou simplement parfois au gré des humeurs. Et les variations terminologiques ne s'arrêtent pas là. C'est en fait tout un réseau de termes qui se trouvent convoqués, où l'on trouve aussi bien les notions d'éventualité, et de supposition, mais encore de réserve voire de restriction, où il sera question d'envisager, ou d'admettre, de déduire ou d'inférer, de conditionner ou d'impliquer.

En droit, toutes ces notions, quoique liées, ne sont pas équivalentes. On pourrait voir dans ces confusions un simple manque de rigueur des descripteurs. Ou l'on pourrait y voir un défaut des notions elles-mêmes, qui seraient trop floues, mal différenciées, voire mal adaptées. Mais l'on peut y voir aussi le symptôme d'une difficulté réelle: de fait, les valeurs qu'il s'agit de décrire sont à chaque fois d'un certain point de vue distinctes et, d'un autre point de vue, globalement équivalentes; et de fait, le terme de condition, y 
compris lorsqu'on l'emploie dans son sens courant (et non dans une acception construite qu'aurait forgé la métalangue des grammairiens), peut effectivement s'appliquer à tous les énoncés concernés,mais pas de la même façon à chaque fois, mobilisant alors des différences que les autres notions convoquées par la tradition permettent d'expliciter.

Pour rendre compte de cette élasticité, je partirai d'une caractérisation de la notion de condition (si ce n'est dans son usage ordinaire, du moins dans son usage métalinguistique, pour regrouper tous les énoncés du champ) qu'une première analyse des énoncés concernés m'a permis d'élaborer (de Vogüé, à paraitre).

Il s'avère que cette notion ne peut être ni réduite à une relation d'implication (il y a des énoncés conditionnels non-implicatifs), ni cantonnée à des énoncés portant sur des situations fictives (il y a des énoncés conditionnels dans lesquels $\mathrm{p}$ est donné comme étant vérifié).

11 J'ai soutenu qu'elle devait être rapportée à une configuration énonciative se caractérisant par quatre propriétés :

- la situation que désigne la protase (Sitp) doit être inscrite dans une classe de possibles;

- la nature qualitative de la propriété $\mathrm{p}$ exprimée par la protase doit constituer un critère déterminant tant dans la sélection de Sitp, que dans la prédication opérée par l'apodose ;

- la prédication opérée par l'apodose doit être une prédication thétique, et non pas catégorique, ce qui signifie que la propriété q n'est pas une propriété attribuée à Sitp : Sitp ne fait que définir le cadre dans laquelle cette prédication thétique est valide ;

- cette prédication d'existence doit impliquer un choix subjectif: le choix d'un sujet qui manifeste son libre arbitre de sujet en prédiquant q plutôt qu'autre-que-q dans le cadre que définit Sitp.

12 La «figure conditionnelle» résulterait donc du montage de trois opérations énonciatives :

(A) : parcours d'une classe de possibles ;

(B) : spécification de Sitp par $\mathrm{p}$;

(C) : prédication existentielle de q engageant un sujet pour qui $\mathrm{p}$ sélectionne q plutôt qu'autre-que-q.

13 Je prétends que cette figure constitue le point commun à tous les énoncés conditionnels, et qu'elle est suffisamment « élastique » pour rendre compte de toutes les variations dont le champ conditionnel est le lieu. Ce sont les mécanismes dont ces variations procèdent qui seront ici examinés.

\section{La façon dont les opérations s'articulent}

Dans le montage décrit ci-dessus, c'est (B) qui détermine l'issue du parcours (A) :

$\left(A^{\prime}\right)$ : Sitp est sélectionné en tant que $p$ le spécifie.

Elle le circonscrit doublement, au cadre que définit Sitp d'une part, au fait que $\mathrm{p}$ spécifie Sitp d'autre part, ces deux composants n'intervenant pas de la même façon dans la prédication opérée.

17 J'ai montré en effet que la prédication en question ne vaut dans un énoncé conditionnel que pour la (ou les) situation(s) particulière(s) considérée(s) et non pas (ou pas nécessairement) pour toute occurrence de $\mathrm{p}$ : dans (5) ci-dessous, il n'est pas dit que la 
malheureuse victime sera frappée dans toutes les situations où elle ouvrira la bouche, mais seulement dans une situation donnée, qui est celle qu'évoque l'énoncé :

(5) S'il ouvre la bouche, je le frappe. ${ }^{6}$$$
\text { conditionnelle : }
$$

(B') : Sitp valide ${ }^{7}$.

(C') : p détermine la sélection par un sujet de q plutôt qu'autre-que-q.

21 Il apparaît dès lors que la constitution de la figure conditionnelle procède de plusieurs nouages : nouage de (A) avec (B), qui produit (A'); puis nouage de (A') avec (C), qui de son côté se dédouble en deux nouages qu'il faut différencier, à savoir celui de ( $\left.A^{\prime}\right)$ avec (B'), et celui de (A') avec ( $\left.C^{\prime}\right)$. Doivent être ainsi articulés parcours de la classe et spécification via $p(A)$ et $(B)$, sélection de Sitp et validation de $q\left(A^{\prime}\right)$ et (B'), spécification via p et sélection $\operatorname{de~} q\left(A^{\prime}\right)$ et $\left(C^{\prime}\right)$.

22 Je montrerai qu'il y a en chacune de ces articulations un jeu possible. C'est ce triple jeu qui nous permettra de rendre compte des déformations auxquelles la figure conditionnelle donne lieu, et de découvrir ce faisant, la façon dont le champ élastique de la condition se trouve structuré.

\section{Conditions initiales et conditions de validation}

On commencera par analyser l'articulation qui s'opère entre sélection de Sitp et validation de $\mathrm{q}\left(\mathrm{A}^{\prime}\right)$ et (B'). Le nouage de ces deux opérations suppose que la situation Sitp sélectionnée soit une situation qui valide q. On voit que la formulation de cette articulation suppose l'utilisation d'une relative : " une situation qui valide $q$ ». On sait qu'en droit une relative est compatible avec (au moins) deux interprétations, l'une dite "prédicative », l'autre dite "déterminative ». Dans la première, la situation est sélectionnée indépendamment (via p), et la relative dit qu'à partir de cette situation q est validé : Sitp fait alors simplement fonction de support de la validation en question. Dans la seconde en revanche, la situation est sélectionnée en tant qu'elle valide q: Sitp est alors déterminée par le fait qu'elle valide q.

S'il est vrai que la figure conditionnelle ne précise pas laquelle de ces deux interprétations choisir, on doit s'attendre à ce que cette figure recouvre deux catégories de conditions fortement différenciées : d'un côté une situation de départ (caractérisée qualitativement), à propos de laquelle q se trouve validé ; de l'autre une situation qui est définie d'emblée en relation à la validation de q, par le fait même qu'elle le valide.

Il se trouve que la notion de condition, telle qu'elle est véhiculée par le mot condition employé dans la langue ordinaire, admet effectivement ces deux interprétations ${ }^{8}$ :

(6) les conditions de vie

(7) les conditions de sa survie. 
Dans (7), le terme désigne ce qui serait nécessaire pour qu'« il » ou « elle » survive. Dans (6), le fait que cette personne vive est acquis, et il s'agit seulement de désigner la façon dont elle vit. L'opposition est extrêmement nette: d'un côté les conditions "pour " qu'elle puisse survivre, de l'autre les conditions «dans lesquelles» elle se trouve vivre, qui sans doute conditionnent sa vie, mais ne conditionnent pas le fait qu'elle vive.

L'on doit par conséquent distinguer entre ce que j'appellerai les " conditions initiales » d'un processus, qui déterminent la nature du processus en question ou la façon dont il va se réaliser, et ce qui serait plutôt les "conditions de validation" d'un processus, qui déterminent la possibilité qu'il ait lieu, et qui sont donc définies à partir de ce processus lui-même, comme ce qui peut être nécessaire, ou ce qui peut être suffisant à son actualisation ${ }^{9}$. On voit que les premières correspondent à la configuration mise en place par la lecture prédicative de la relative, tandis que les secondes s'inscrivent dans la lecture déterminative, où la situation sélectionnée est définie par le fait que q s'y réalise.

D'un côté donc les conditions « dans lesquelles » il va s'avérer que q se réalise, de simples conditions initiales pour q; de l'autre, les conditions "pour" que q se réalise, des conditions de validation. Lorsque le terme de condition est employé pour gloser les énoncés conditionnels, il l'est en fait, selon les cas, dans l'une ou l'autre de ces acceptions. Les énoncés construits avec la locution à condition que réfèrent clairement à une condition de validation: ils explicitent ce qu'il faut pour que q se réalise. En revanche, dans un énoncé comme (8) par exemple, p n'est plus donné comme une condition pour que q se réalise (si tant est que la formule puisse avoir un sens pour ce cas où q est une question) :

(8) A supposer qu'il soit là, qu'est-ce que tu fais ?

Il demeure que dans une glose de (8), on pourra tout à fait utiliser le terme de condition, mais cette fois pour désigner les conditions initiales, qui déterminent $q$ (ce que tu vas faire), et même la façon dont la question de q peut se poser.

Cette distinction travaille la figure relationnelle de la condition, travaille aussi la notion ordinaire de condition, et on la voit remodeler la valeur même des énoncés conditionnels. L'exemple précédent et sa glose (à condition que / à supposer que) indiquent, en outre, qu'elleva dépendre crucialement du marqueur qui se trouve utilisé pour construire l'énoncé conditionnel: on aurait ainsi affaire à un premier clivage au sein du champ conditionnel.

Pour départager les marqueurs qui introduisent une condition de validation deceux qui introduisent une condition initiale, on peut recourir aux gloses en s'interrogeant sur la valeur qu'y prendra le terme de condition. Mais il est possible aussi d'élaborer des tests, tenant compte des caractéristiques propres à chaque catégorie. On en propose ici deux.

Considérons l'énoncé (9) :

(9) Si tu l'invites maintenant, elle t'en voudra quand même de ne pas l'avoir invitée dès le départ.

La présence de quand même dans l'apodose indique une forme d'opposition entre $\mathrm{p}$ et $\mathrm{q}: \mathrm{q}$ est validé malgré $p$, c'est-à-dire alors même que $p$ aurait pu avoir pour conséquence nonq. Dans une telle configuration, p ne saurait être tenu comme conditionnant la possibilité de $\mathrm{q}$, puisque $\mathrm{p}$ tend au contraire à aller contre q. On s'attend donc à ce qu'un marqueur de condition de validation soit incompatible avec ce type de contexte propositionnel. Par exemple les séquences rassemblées en (10) paraissent irrémédiablement mal construites :

(10) *A condition que tu l'invites maintenant, elle t'en voudra quand même de ne pas l'avoir invitée dès le départ. 
*Pour peu que tu l'invites maintenant, elle t'en voudra quand même de ne pas l'avoir invitée dès le départ.

\section{(10) sont strictement impossibles :}

(14) *A condition que tu l'invites ou (comme) à condition que tu ne l'invites pas, il viendra.

*Pour peu que tu l'invites, ou (comme) pour peu que tu ne l'invites pas, il viendra. *Dès l'instant que tu l'invites ou (comme) dès l'instant que tu ne l'invites pas, il vient.

41 deux tests, aux résultats convergents, permettent d'envisager un classement de l'ensemble des marqueurs du champ en deux catégories : un tel classement est proposé dans les colonnes du tableau 1 inséré en annexe. Si l'on parcourt la liste des marqueurs classés dans le registre de la condition de validation, ils indiquent tous ce qu'il faut ou du moins ce qu'il suffit pour que q puisse être valide ; et ils ont pour effet interprétatif que $p$ paraisse en quelque sorte "dirigé» vers une conclusion (ce que marquent les prépositions du, dès, pour, à, sous, ainsi queles participes moyennant et pourvu). En revanche, les marqueurs classés dans le registre de la condition initiale, indiquent que l'on envisage une circonstance ("soit une situation vérifiant $\mathrm{p}$ »: d'un point de vue formel, la prédominance des groupes prépositionnels en dans serait le symptôme de ce " changement de cadre »), et que cette circonstance «donne » q, mais de manière cette fois contingente ( tu prends $p$, il se trouve que tu as $q$ »). 

introduction. Lorsqu'il est antéposé, les tests le placent a priori plutôt du côté de la condition initiale (comparer (15) avec (8)). Et l'on perçoit effectivement que la valeur première de si va être de définir une circonstance possible, pour voir ce qui s'y avère vrai. Pourtant si peut aussi prendre l'autre valeur, lorsqu'il apparaît en position prédicative comme en (16), où j'ai montré qu'il confère alors à $\mathrm{p}$ le statut de condition nécessaire (donc éminemment conditionnante voir (de Vogüé 1987b)) :

(15) S'il vient, qu'est-ce que tu fais?

(16) J'irai s'il m'invite. ${ }^{11}$

Si ne met donc pas en péril la division entre conditions initiales et conditions de validation. Simplement, il est suffisamment neutre, ou suffisamment souple lui-même, pour passer d'une catégorie à l'autre.

\section{Eventualités et suppositions}

La seconde articulation que nous étudierons est celle qui se joue dans l'opération même de sélection de Sitp (A'), à savoir celle où la spécification de Sitp par $\mathrm{p}$ détermine l'issue du parcours effectué sur la classe des possibles.

Sitp est sélectionné en tant que $\mathrm{p}$ le spécifie. Cela aussi peut s'interpréter de deux manières. Il s'avère en effet que la classe des possibles sur laquelle la sélection s'opère peut être de différentes natures : classe de situations possibles, avec d'autres Sit que Sitp, ou classe d'alternatives possibles, avec cette fois autre-que-p comme alternative possible à p. On parcourt donc soit des situations, soit des propriétés. Si on parcourt des situations, $\mathrm{p}$ fait fonction de critère discriminant pour écarter les situations où il ne s'instancie pas. Si on parcourt des propriétés, on ne sélectionne Sitp que dans la mesure où l'on sélectionne $\mathrm{p}$, et où Sitp se trouve instancier $\mathrm{p}$.

Autrement dit, dans le premier cas p est le moyen de la sélection de Sitp : on sélectionne " grâce à » p, ou à l'aide de p. Dans le second, p, ou plutôt le fait que p spécifie Sitp, est la raison de la sélection de Sitp : c'est «à cause de » p, parce que p spécifie Sitp, que Sitp est sélectionné. Ce qui paraît n'être qu'un jeu de mots (grâce / à cause) mais qui est en fait un jeu sur la nature des circonstances de la sélection (moyen/raison: double lecture de la relation entre « sélectionné » et « en tant que » dans $\left.\left(\mathrm{A}^{\prime}\right)\right)$, met en cause non seulement la nature de la classe où s'opère cette sélection (classe de situations, classe de propriétés), mais le mouvement même dont cette sélection procède: d'un côté une simple discrimination avec $\mathrm{p}$ comme critère, de l'autre un mouvement double, avec d'abord sélection de $\mathrm{p}$, puis instanciation de $\mathrm{p}$.

Est en cause aussi le type de relation qui va être établie entre $p$ et Sitp. Le fait que $p$ spécifie Sitp signifie en effet dans le premier cas que $p$ marque la spécificité de Sitp dans la classe, distingue Sitp des autres (le discrimine). Dans le second cas, la spécification en question doit s'entendre comme une définition : $p$ est ce qui va permettre de définir Sitp. On a des situations et on en discerne une grâce à une propriété qu'elle a et qui, dès lors, la caractérise par rapport aux autres; ou on a une propriété, et cette propriété détermine une situation, parce qu'elle spécifie ce qui peut singulariser la situation en question ${ }^{12}$. Dans le premier cas, $\mathrm{p}$ fait figure en quelque sorte d'étalon, et on mesure la conformité des situations à cet étalon. Dans le second p est un prédicat, susceptible en tant que tel de représenter des situations (d'en donner une représentation). 
Or, on trouve bien dans le champ fluctuant des énoncés conditionnels des cas où la condition est une éventualité, évoquant une situation possible que $\mathrm{p}$ sert à sélectionner parmi d'autres éventualités. Et l'on trouve des cas où la condition est une supposition évoquant cette fois une propriété, et où l'énoncé va alors mesurer les conséquences de cette propriété sur la situation qui l'instancierait. (17), qui envisage une situation qui pourrait advenir, ressortit au premier cas, tandis que (18), qui prétend donner les implications d'une proposition dont il s'agit de prendre la mesure, ressortit au second cas :

(17) S'il entre, tu te tais.

(18) S'il est passé ici hier, il est déjà loin.

51 On voit que la différence peut être rendue à nouveau par une glose : « s'il arrive que » pour (17), « s'il est vrai que » pour (18).

La question est de savoir si cette opposition peut rendre compte des différences entre les locutions qui se partagent le champ de la condition. Il semble que ce soit le cas, quand bien même si s'avère à nouveau être neutre par rapport à l'opposition en question. Pour montrer le clivage que ce critère de différenciation opère, on utilisera cette fois encore deux tests.

53 Pour diagnostiquer les marqueurs propres à exprimer la supposition, la configuration (18) fournit un test assez éclairant, bien qu'il nécessite de recourir à la glose et ne produise pas directement des inacceptables : «il est passé ici hier » peut toujours être interprété non plus comme renvoyant à une assertion (envisagée ou reprise) dont on mesure les conséquences, mais comme renvoyant à ce qui aurait pu se passer « hier ». On vérifiera que c'est ce qui se produit dans les énoncés (20) qui évoquent une situation, ce qui peut avoir eu lieu « hier ", tandis que dans (19) p conserve le statut de proposition à évaluer : et de fait, dans (19), on peut, après la protase, insérer la glose « si c'est vrai », tandis que dans (20) la glose sera « si cela a eu lieu » :

(19) A supposer qu'il soit passé ici hier, il est déjà loin.

Dans l'hypothèse où il est passé ici hier, il est déjà loin. 
Sous réserve qu'il soit passé ici hier, il est déjà loin.

Pour autant qu'il soit passé ici hier, il est déjà loin.

(20) Il est passé ici hier, (si cela a eu lieu / ? ? si c'est vrai) il est déjà loin.

Au cas où il serait passé ici hier, (si cela a eu lieu / ? ? si c'est vrai) il serait déjà loin.

A condition qu'il soit passé ici hier, (si cela a eu lieu /?? si c'est vrai) il est (forcément) déjà

loin.

Pour peu qu'il soit passé ici hier, (si cela a eu lien / ? ? si c'est vrai) il est déjà loin.

Ce premier paradigme indique en particulier que l'opposition éventualité / supposition est l'un des paramètres qui différencie à condition que et sous réserve que. Ces deux locutions paraissent pourtant l'une et l'autre exprimer une réserve, et pourraient sembler ne se distinguer que sur un plan strictement stylistique. Ce qui ressort de notre analyse est que la réserve concerne un type de situation dans un cas, une proposition dans l'autre.

Le second test est plus difficile encore à construire : toute description d'une situation éventuelle peut aussi s'interpréter comme une proposition dont on mesure la pertinence pour décrire la situation en question. Ainsi à supposer qu'il entre peut s'interpréter comme signifiant "à supposer que dans la situation considérée, il soit vrai qu'il entre»: l'événement (auquel entrer renvoie "naturellement » en tant que verbe d'achèvement) a été réinterprété comme une propriété. Dans la mesure où il est plus difficile de réinterpréter une propriété (s'exprimant dans une proposition) comme un événement (introduisant une situation), on proposera donc plutôt d'observer une configuration où apparait en protase un prédicat relevant de la catégorie aspectuelle de la propriété (et non de l'achèvement ou de l'accomplissement selon les grilles de Vendler $\left.{ }^{13}\right)$ : si l'on a affaire à une construction marquant l'éventualité, celle-ci doit être capable de transformer la propriété en événement, le prédicat s'interprétant alors comme référant à l'avènement de la propriété en question.

Considérons par exemple (21):

(21) S'il l'aime, il l'invitera.

(21) est compatible avec une interprétation dans laquelle on s'interroge sur la question de savoir s'il est ou non vrai qu'actuellement il l'aime: on est dans le registre de la supposition. Mais (21) est compatible aussi avec une autre interprétation, dans laquelle on s'interroge sur les effets possibles d'une situation où, rencontrant quelqu'un, il se mettrait à l'aimer : il s'agit alors d'une éventualité (une situation nouvelle, autre), et on peut gloser l'énoncé par «si cela arrive ». Or une telle glose, insérée après la protase, ne paraît pas compatible avec les locutions rassemblées en (19) :

(22) A supposer qu'il l'aime, ( ? ? si cela arrive / s'il est vrai qu'il l'aime maintenant),

il l'invitera.

Dans l'hypothèse où il l'aimerait ( ? ? si cela arrivait / s'il était vrai qu'il l'aime), il

l'inviterait.

Sous réserve qu'il l'aime ( ? ? si cela arrive / s'il est vrai qu'il l'aime actuellement), il

l'invitera.

Pour autant qu'il l'aime ( ? ? si cela arrive / s'il est vrai qu'il l'aime actuellement), il

l'invitera.

On comparera (22) avec (23), où l'interprétation événementielle, avec l'amour advenant dans la situation envisagée, est possible à construire :

(23) Il l'aime (s'il arrive qu'il se mette à aimer la personne qu'il rencontre), il

l'invite.

Au cas où il l'aimerait (s'il arrivait qu'il se mette à l'aimer), il l'inviterait.

A condition qu'il l'aime (s'il arrive qu'il se mette à l'aimer), il l'invitera.

Pour peu qu'il l'aime (s'il arrive qu'il se mette à l'aimer), il l'invitera. 
vanche, dans les énoncés (23), la glose "s'il est / était vrai qu'il l'aime » ne semble pas être acceptable, hormis dans le jargon d'un sémanticien vériconditionaliste s'obstinant à ramener à la problématique de la vérité toute proposition, y compris celles qui réfèrent à des événements.

Il semble donc bien que l'on ait là une nouvelle base de classement, venant croiser le classement en conditions initiales et conditions de validation de la section précédente. On dispose d'ailleurs d'un dernier critère pour distinguer entre les deux catégories ici proposées. Les suppositions n'évoquent pas une situation mais une proposition susceptible de s'appliquer à quelques situations. Dès lors, si les éventualités doivent nécessairement porter sur une situation distincte de la situation actuelle, les suppositions peuvent quant à elles porter sur la situation actuelle : étant donnée la situation actuelle, on se demande ce qui se passerait, si cette situation actuelle avait la propriété p (⿳亠㐅子 supposer qu'il l'aime = « à supposer qu'il soit actuellement vrai qu'il l'aime »).

61 Cela signifie que ce que les suppositions visent à mesurer est en définitive le degré d'actualité d'une proposition: les chances qu'elle peut avoir d'être actuelle, ou de s'actualiser. Et il s'avère au demeurant possible de classer les marqueurs de supposition selon le plus ou moins grand degré d'actualité qu'elles reconnaîtront à p (à supposer que construit une proposition hors actualité, tandis que pour autant que construit une proposition donnée comme pouvant être actualisée).

Les éventualités en revanche sont construites comme des situations autres : on n'est donc plus dans une problématique de l'actualité, il ne s'agit plus d'évaluer si p est valide dans la situation actuelle, celle-ci étant dans ce cas littéralement hors jeu. Seul peut s'évaluer le degré de possibilité de cette situation, selon qu'elle est donnée comme envisageable (concevable: pondération sur le possible = "peut » arriver), ou comme simplement envisagée (équipondération entre "peut » et "peut ne pas »). Là encore un classement des marques concernées peut être mis en place: avec pour peu que, l'éventualité est donnée comme étant de l'ordre de l'envisageable (pas nécessaire sans doute, mais néanmoins possible : il en faut «peu »), tandis qu'avec un coup que, elle est rapportée au domaine de la pure contingence.

\section{Bilan d'un double clivage}

63 On arrive au bout du compte, en distinguant 5 degrés d'actualisation, et 5 modalisations $\mathrm{du}$ possible ${ }^{14}$, à proposer un classement ordonnant une vingtaine des marques de condition, selon les critères du type de condition d'une part, du statut d'éventualité ou de supposition de la protase d'autre part : ce classement est donné dans le tableau 1.

Il n'est pas définitif: les critères pour l'établir sont plus ou moins assurés selon les cas (plus pour ce qui concerne l'opposition condition initiale /condition de validation, moins pour ce qui concerne les degrés, pour lesquels on se contente de s'en remettre à l'intuition) ${ }^{15}$, l'ensemble des marqueurs n'est pas couvert ${ }^{16}$. Il demeure que le grand désordre de la catégorie trouve là un début de structuration, permettant de décrire avec quelque précision la valeur de chacun des marqueurs concernés. 


\section{La particularité de si} à chacune des déformations de la figure conditionnelle explicitées dans les sections 2,3 et 4. dialectiques, ou explicatives, dont on a vu qu'elles sont extérieures au champ de la condition, mais d'autres encore qui, tout en restant dans le champ de la condition, ne se retrouvent pas avec les autres marqueurs. Simplement, celles-là sont moins manifestes, et plus difficiles à mettre en évidence : elles participent de la confusion générale décrite dans la première section, où la quasi-synonymie prévaut et où l'équivalence menace toute les distinctions.

67 Je propose de rendre compte de la spécificité de si en montrant que ce marqueur exploite non seulement le jeu laissé par les deux articulations qui viennent d'être analysées, mais aussi celui que laisse la troisième articulation, entre spécification via $p$ et sélection de $q$ telle qu'elle s'exprime en $\left(C^{\prime}\right)$ : $p$ détermine la sélection de q pour un sujet.

\subsection{Les deux lectures de $\left(C^{\prime}\right)$}

Considérons en effet ( $\left.C^{\prime}\right)$. A nouveau le nouage peut s'effectuer de deux manières, selon la façon dont le sujet en question va s'inscrire dans la sélection opérée : ou bien c'est lui qui sélectionne $q$, "à cause de " $p$, et $p$ n'est par conséquent que la raison de cette sélection où le libre arbitre du sujet se manifeste; ou bien, ce libre arbitre réside dans le fait d'estimer que $\mathrm{p}$, en lui-même, sélectionne $\mathrm{q}, \mathrm{p}$ faisant alors figure de moteur de la sélection. On peut résumer l'alternative à l'aide des deux formules suivantes :

(C1') Vu p, S sélectionne q.

(C2') Selon S, p sélectionne q.

On pourrait considérer que ces deux formules, à défaut d'être équivalentes, aboutissent du moins à des résultats équivalents : c'est bien la manifestation de la quasi-synonymie qui règne sur l'expression de la condition. Elles n'en sont pas moins crucialement distinctes, sur le plan formel d'abord (dans les deux cas, $\mathrm{p}$ et $\mathrm{S}$ interviennent dans la sélection, mais ils n'interviennent pas au même endroit ou au même titre), sur le plan des opérations mises en œuvre ensuite. Dans les deux cas, il faut que $S$ ait perçu et évalué $p$ (en ait pris connaissance et en ait pris la mesure), mais dans le premier cette évaluation justifie et donc précède la sélection où $S$ témoigne de son libre arbitre, alors que dans le second les deux opérations ne sont en fait pas dissociées: l'évaluation de p consiste à estimer que $\mathrm{p}$ sélectionne $\mathrm{q}$

\subsection{Ce qui se joue dans la configuration (C2') : le concept d'hypothèse et la catégorie des énoncés « relationnels »}

Quelles seront alors les caractéristiques d'un énoncé s'inscrivant dans cette seconde configuration?

La sélection de q n'est plus rapportée au libre arbitre du sujet, mais à une nécessité qui serait attachée à $p$ (du point de vue d'un sujet) : on aura donc affaire à des énoncés dans 
lesquels la relation entre $\mathrm{p}$ et $\mathrm{q}$ est donnée comme une relation objective, n'impliquant aucune subjectivité (la subjectivité est ailleurs : dans le choix de cette relation). Dans la plupart des énoncés considérés jusque-là, $p$ donne $q$ dans la mesure où un sujet choisit qu'il donne q; ici le sujet soutient que $\mathrm{p}$ lui-même, de par sa dimension qualitative propre, donne q. Il faut alors qu'il y ait une relation sinon sémantique, du moins cognitive, associant $\mathrm{p}$ et $\mathrm{q}$ de manière intrinsèque, en tant que qualités. Ou il faut au moins que la relation soit donnée comme valant pour toute occurrence de $\mathrm{p}$, sans exception particulière possible (sans liberté de choix possible), donc de manière générique. On voit que ce portrait correspond très exactement à la configuration à laquelle on veut parfois, de manière abusive, cantonner le domaine de la condition : une relation qualitative générale entre deux propositions, alors que l'implication conditionnelle se réduit le plus souvent à un simple déterminisme contingent, limité à une situation particulière et fondé sur le libre arbitre d'un sujet.

On a dit par ailleurs que c'était dans le cadre de l'évaluation qui est faite de p par un sujet que $\mathrm{p}$ se trouvait ainsi rattaché à $\mathrm{q}$. Autrement dit, l'énoncé tout entier consiste dans cette évaluation, alors qu'elle n'intervenait jusque-là que pour fonder q. On évalue une proposition, on évalue ses conséquences, ce qu'elle sélectionne, et c'est la relation entre $p$ et q qui donne la mesure de cette évaluation. Par conséquent, la protase n'est pas simplement une éventualité, ou une supposition servant de cadre à la prédication opérée : l'objet de l'énoncé est d'évaluer la proposition qu'exprime cette protase.

Or, le fait qu'elle soit soumise à évaluation est bien ce qui distingue une hypothèse d'une pure éventualité ou supposition. Lorsque le terme d'hypothèse est employé (hors du champ de la grammaire), que ce soit dans le raisonnement scientifique, ou dans le discours ordinaire de quiconque cherchant à trancher une question, c'est pour désigner une proposition dont on ne sait s'il est vraie ou fausse, mais que l'on choisit de prendre momentanément pour vraie, afin de pouvoir mesurer ce qu'elle implique, et décider par là de sa vérité : une hypothèse est à évaluer, «faire " cette hypothèse permet de se donner les moyens de l'évaluer, et on l'évalue précisément en prenant la mesure de ce qu'elle sélectionne. Les énoncés entrant dans la configuration (C2') sont donc des énoncés dans lesquels la protase fait fonction d'hypothèse. Et c'est pour cette raison que la relation entre $p$ et $q$ est une relation nécessaire et qualitative : c'est à ce prix que s'évalue une hypothèse.

Reste une dernière particularité de cette configuration: $\mathrm{p}$ étant soumis à évaluation, la relation entre $p$ et $q$ est ce que l'énoncé exprime, ce qu'il propose pour évaluer $p$. C'est dire que cette relation passe au premier plan: au lieu d'être seulement mobilisée pour fonder la prédication de $q$, elle constitue directement ce que l'énoncé affirme relativement à p. (C2') produit par conséquent une catégorie d'énoncés que l'on pourrait qualifier de "relationnels", par opposition aux autres qui sont "prédicatifs»: les premiers ont pour objet de poser une relation entre $p$ et $q$, tandis que les seconds se contentent d'asserter $\mathrm{q}$, dans le cadre défini par Sitp et caractérisé par $\mathrm{p}$.

La protase $p$ fait fonction d'hypothèse, la relation entre $p$ et $q$ est nécessaire et qualitative, et l'énoncé a pour contenu d'exprimer cette relation: la configuration (C2') s'avère par conséquent correspondre à la valeur prototypique de la condition, celle que l'on croit à tort pouvoir étendre à tout le champ de la condition, alors qu'elle n'est qu'une interprétation possible de la figure conditionnelle. 


\subsection{Les valeurs (C2') de si}

Si a des emplois qui relèvent de (C2'). Cela explique son caractère prototypique. Mais c'est aussi ce qui fait sa spécificité : ces emplois lui sont propres.

Si a des emplois « relationnels », exprimant directement la relation entre p et q, en faisant abstraction de la situation concrète Sitp que $\mathrm{p}$ singularise. C'est lui que l'on utilisera pour justifier un énoncé conditionnel " prédicatif ", et énoncer la relation rattachant pà q qui légitime la prédication opérée. Ainsi est-ce si qui sera utilisé dans le dialogue suivant, lorsque $\mathrm{X}$ est amené à expliciter (d'une manière qui n'est qu'apparemment circulaire) le savoir dont il s'est autorisé pour asserter le premier énoncé :

(24) $\mathrm{X}$ : A supposer qu'il pleuve, tu le trouveras au cinéma.

$\mathrm{Y}:$ Comment le sais-tu?

$\mathrm{X}$ : Je sais simplement que s'il pleut, il sera au cinéma.

Et c'est si encore qui sert de manière générale à poser une relation d'implication générique. L'exemple qu'analyse Frege dans Sens et dénotation en est une illustration :

(25) Si un nombre est compris entre 0 et 1, son carré est compris entre 0 et 1 .

Un tel énoncé ne porte pas sur un nombre particulier, aussi peu défini soit-il. Il ne porte même pas sur l'ensemble des nombres compris entre 0 et 1 , bien que ce qu'il dit concerne cet ensemble et permette de lui attribuer la propriété d'avoir un carré compris entre 0 et 1. Dans le contexte où Frege propose de l'employer, celui d'un discours mathématique où sont examinées des propriétés mathématiques et où sont énoncées des lois, cet énoncé évalue les conséquences d'une hypothèse : il porte non pas sur un ensemble mais sur une hypothèse. Et il prend la mesure de cette hypothèse en énonçant une relation: (25) exprime une relation entre propositions.

Sans doute l'énoncé pourrait-il avoir une autre interprétation, où il s'agirait bel et bien d'envisager une situation, celle que décrit $p$, pour alors prédiquer $q$, en se fondant sur $p$. On peut forcer cette lecture, en insérant par exemple dans ce cas-là après la protase. Mais ce n'est pas son interprétation lorsqu'il exprime une loi des nombres.

81 En revanche ce serait la seule interprétation possible avec tout autre marqueur: on entend clairement la distinction si l'on compare (25) avec (26) :

(26) A supposer (pour peu que, à condition que, etc.) qu'un nombre soit compris entre 0 et 1 , son carré est compris entre 0 et 1 .

(26) évoque une situation, celle d'un nombre quelconque qui serait compris entre 0 et 1 , et s'engage alors sur q, qui concerne le carré de ce nombre. Partant d'une proposition, (26) énonce une autre proposition, et non pas le lien entre les deux, qui lui est exprimé par (25).

33 Si a aussi un autre emploi, qui est parfois évoqué dans les descriptions, quand il sert à opérer ce que l'on appelle une déduction :

(27) S'il appelle, c'est qu'il a quelque chose à demander.

De tels énoncés cumulent en quelque sorte deux opérations: on envisage bien une situation, mais la proposition qui caractérise cette situation est alors évaluée, et c'est à ce qu'elle implique qu'elle est évaluée. L'apodose n'exprime pas ce qui est vrai de la situation en question, mais ce qu'implique cette situation, c'est-à-dire ce que l'on peut déduire de la protase. Celle-ci est donc bien prise comme une hypothèse, quoiqu'elle décrive aussi une situation, et l'énoncé dit à la fois que q doit être vrai, et que de $\mathrm{p}$ s'infère q. 

uns n'acceptent tout simplement pas une apodose en c'est que; d'autres perdent avec c'est que leur valeur conditionnelle; dans d'autres enfin, q ne vaut plus comme une déduction mais comme une explication :

(28) *Pourvu que (à condition que, ...) il appelle, c'est qu'il a quelque chose à demander.

(29) Du moment (dès l'instant, dès lors) qu'il appelle, c'est qu'il a quelque chose à te demander ( = étant donné que).

(30) A supposer que (au cas où,...) il appelle, c'est qu'il a quelque chose à demander.

Il y a encore un emploi que l'on pourrait décrire comme métalinguistique, où il s'agit de spécifier le sens des termes impliqués dans $\mathrm{p}$ :

(31) Si c'est un voilier, cela signifie que c'est un bateau.

A nouveau, les autres marqueurs sont incompatibles avec ce type d'explicitation de $\mathrm{p}$ : dans la plupart des cas, le passage de voilier à bateau donne un énoncé que l'on qualifiera au mieux de déconcertant, parce que la tautologie qui s'y exprime n'est pas compatible avec le type de prédication existentielle que la locution « promet » (? ? Pour peu que ce soit un voilier, c'est un bateau); dans les autres, il est concevable que cette prédication d'existence consiste à tirer les conséquences y compris sémantiques de $\mathrm{p}$ (Du moment que c'est un voilier, c'est un bateau), mais q ne vaut pas alors comme une prédication métalinguistique, et c'est la formule cela signifie que qui paraît problématique ( ?? Du moment que c'est un voilier, cela signifie que c'est un bateau).

Et puis il y a le si thématique, dans lequel $\mathrm{p}$ est pris pour acquis, et dont l'enjeu est de prendre la mesure de cet acquis, en explicitant ce qui s'en infère (ce qu'il signifie) :

(32) Il est chez Paul, et s'il est chez Paul, il ne peut pas avoir d'ennuis. (glose en vu que)

Avec les autres marqueurs, ou bien la protase retrouve un caractère fictif (A supposer qu'il soit chez Paul, il ne peut pas avoir d'ennuis), ou bien on sort du champ de la condition, parce que le marqueur prend un sens soit causal soit temporel (Dans la mesure où il est chez Paul, il ne peut pas avoir d'ennuis).

90 Il y a le si de reprise, dans lequel $\mathrm{p}$ reprend l'assertion d'autrui, non pas pour l'interroger, mais pour en tirer les conséquences «naturelles » (ce que $p$ sélectionne), la vérité de $p$ n'étant par conséquent pas prise pour acquise, mais étant néanmoins admise (au sens où elle n'est pas mise en cause) :

(33) S'il fait beau, on n'a qu'à aller se promener.

(glose en puisque)

91 A nouveau les autres marqueurs ou bien seront incompatibles avec cette non mise en cause de $\mathrm{p}$, ou bien n'auront plus une valeur conditionnelle.

Il y a enfin les emplois de si avec l'imparfait, que seul si autorise à prendre cette valeur non révolue. J'ai soutenu dans une étude indépendante relative aux valeurs de l'imparfait ${ }^{17}$ que les énoncés produits se répartissaient en deux catégories distinctes, l'une proprement contrefactuelle qui tire les conséquences invraisemblables de $\mathrm{p}$ pour mieux l'invalider, l'autre simplement plus fictive qu'au présent, où il s'agit d'envisager $p$, non avéré mais néanmoins concevable, pour dire ce qui en adviendrait :

(34) Sij'étais riche, je ne passerais pas ma vie à travailler (voyons!).

(35) Si je gagnais au loto, je m'achèterais une villa en bord de mer. 
Dans tous ces cas, p, vrai ou faux, est pris comme une hypothèse qu'il s'agit d'évaluer ; on l'évalue en spécifiant ce qu'elle sélectionne; et l'énoncé vise par conséquent à faire entendre la relation qui rattache $\mathrm{p}$ à $\mathrm{q}: \mathrm{q}$ n'est jamais à proprement parler asserté, comme l'indiquent les marques modales (ne peut pas, n'a qu'à, conditionnel) qui le caractérisent ; le locuteur se contente de le présenter comme s'inférant de p.

Par ailleurs, il y a les trois valeurs problématiques de si mentionnées dans l'introduction, qui sont hors du champ de la condition, maisqui s'intègrent parfaitement dans la configuration que décrit (C2').

Dans les trois cas, l'énoncé a pour objet d'évaluer $\mathrm{p}$ : le dialectique évalue, négativement, la valeur de vérité de $\mathrm{p}$; le concessif évalue ce que peut signifier et impliquer $\mathrm{p}$ (qu'est-ce qu'implique qu'il soit riche? que peut signifier l'assertion selon laquelle il est riche? jusqu'où peut-on admettre qu'elle est vraie? riche à quel point ?) ; l'explicatif évalue la raison d'être de $\mathrm{p}$ et la façon dont on peut l'interpréter (ne te fais pas d'illusion sur le fait qu'il soit riche, c'est seulement qu'il a gagné au loto, cela ne veut rien dire de plus).

Dans les trois cas, $\mathrm{q}$ donne la mesure de $\mathrm{p}$ (donne $\mathrm{p}$ comme faux dans le dialectique, relativise $\mathrm{p}$ dans le concessif, minimise ou banalise $\mathrm{p}$ dans le cas de l'explicatif).

Dans les trois cas, la relation entre p et q est donnée comme objective : sans doute s'agit-il d'exprimer mon point de vue sur $\mathrm{p}$ (faux ou du moins suspect dans le dialectique, tout relatif dans le concessif, banalisable dans l'explicatif), mais je ne suis pour rien dans la relation entre $p$ et $q$, c'est-à-dire dans le fait que $p$ se trouve prendre la forme $q$ (sélectionne q).

Dans les trois cas enfin, cette relation constitue bien le contenu (ou du moins l'un des contenus comme on va le voir) de l'énoncé : il s'agit de poser que p prend la forme q.

Sans doute la relation entre $p$ et $q$ n'est-elle alors pas une implication, mais on a vu que cela n'avait rien de nécessaire. Sans doute $\mathrm{p}$ est-il alors donné comme étant soit vérifié (l'explicatif), soit avalisé (le concessif), soit remis en cause (le dialectique), mais les exemples précédents prouvent que le caractère non fictif de $\mathrm{p}$ ne suffit pas à retourner la figure conditionnelle et à bannir l'énoncé du champ de la condition : il n'est pas exigé que p soit dépourvu de valeur de vérité.

Si (2-4) ne sont pas des énoncés conditionnels, ce doit donc être pour d'autres raisons : en tout état de cause, et aussi paradoxal que cela puisse paraître, la figure conditionnelle les prévoit.

\subsection{La façon dont s'organisent les valeurs hypothétiques de si}

Les jeux sur la valeur de vérité de $\mathrm{p}$, qui s'observent à la fois dans les exemples où le caractère conditionnel de l'énoncé n'est pas remis en cause (la glose «dans ces conditions » reste de mise) et dans ces trois cas problématiques, peuvent s'interpréter comme les effets des diverses stabilisations de $\mathrm{p}$ dans la classe de possibles que la figure conditionnelle mobilise. On a déjà vu ces effets se manifester dans le cas des éventualités et dans celui des suppositions : de la même façon qu'une éventualité ou une supposition peuvent être données comme plus ou moins possible ou plus ou moins actualisable, il est légitime qu'une proposition soumise à évaluation soit donnée comme plus ou moins vérifiable. 


\section{BIBLIOGRAPHIE}

CULIOLI, A. (1990), Pour une linguistique de l'énonciation, tome 1, Paris, Ophrys.

CULIOLI, A. (1999), Pour une linguistique de l'énonciation, tomes 2 et 3, Paris, Ophrys.

de CORNULIER, B. (1985), Effets de sens (chapitre 4 « L'énonciation conditionnelle »), Paris, Minuit.

DELAVEAU, A. (1990), La conjonction si dans ses emplois interrogatif et conditionnel en français moderne , Thèse de doctorat d'Etat. Université de Paris VII.

DOSTIE, G. (1987), « Etude sémantique de 4 connecteurs conditionnels : à condition que, pourvu que, en autant que et d'abord que ", Le Français Moderne, 87, 3/4, pp. 174-203.

DUCROT, O. (1972), Dire et ne pas dire (chapitre 6 « Supposition et présupposition »), Paris, Hermann.

FEUILLET, J. (1993), « L'hypothèse », in Subordinations / Subordination, CERLICO, 6, pp. 67-96.

FOURNIER, R. et LEARD, J.-M. (1985), «Implications, conditions, hypothèses : perspectives pour l'analyse du système », Revue québécoise de linguistique, IV, 4, pp. 121-131.

FREGE, G. (1892), « Sens et dénotation », in Ecrits logiques et philosophiques (1971), Paris, Le Seuil, pp. 102-126. 
JAYEZ, J. et REBOUL, A. (1990), « Si j'aurais su, j'aurais mis Paris en bouteille : contrefactualité et inférence », Cahiers de linguistique française, 11, pp. 49-75.

PAILLARD, D. et de VOGÜÉ, S. (à paraitre), Altérité et déformation, Paris, Ophrys.

PIOT, M. (1986), « Conjonctions de subordination et problèmes de classification », in Grammaires et histoire de la grammaire. Recueil d'études en hommage à la mémoire de Jean Stéfanini, Amsterdam, Benjamins.

RIEGEL, M., PELLAT, J.-C. et RIOUL, R. (1994), Grammaire méthodique du français, Paris, PUF.

ROUSSEAU, A. (1993), «Implication logique et subordination », in Subordinations / Subordination, CERLICO,6, pp. 233-254.

VENDLER, Z. (1957), « Verbs and times », repris dans Z. VENDLER (1967), Linguistics in Philosophy, Ithaca, Cornell University Press, pp. 97-121.

VISCONTI, J. (1994-1995), « Entre hypothèses et conditions. Sur la sémantique des connecteurs conditionnels complexes ", Cahiers de linguistique française, 15, pp. 77-93.

de VOGÜÉ, S. (1987a), « La conjonction si et la question de l'homonymie », BULAG 13,Université de Besançon, pp. 105-189.

de VOGÜÉ, S. (1987b), « Si, la condition nécessaire et la condition suffisante »,in Actes du colloque sur l'implication dans les langues naturelles et dans les langages artificiels, Université de Strasbourg, 1985, Paris, Klincksieck.

de VOGÜÉ, S. (1993), « Des temps et des modes », Le Gré des Langues, 6.

de VOGÜÉ, S. (1995), « L'effet aoristique », in J. BOUSCAREN, J.-J. FRANCKEL et S. ROBERT (eds), Langues et langage. Problèmes et raisonnement en linguistique. Mélanges offerts à Antoine Culioli, Paris, PUF.

de VOGÜÉ, S. (à paraître), « L'épilangue au pied de la lettre : à propos du concept grammatical de condition », Le Gré des Langues, 16.

WIMMER, C. (1980), « Le système de si en français moderne », Travaux de linguistique et de littérature, 18 (1), pp. 98-116.

\section{ANNEXES}

\section{TABLEAU 1}

\begin{tabular}{|c|c|c|c|}
\hline & & CONDITIONS INITIALES & $\begin{array}{l}\text { CONDITIONS DE } \\
\text { VAL }\end{array}$ \\
\hline SUPPOSITIONS & $\begin{array}{l}\text { Actualisable } \\
\text { Actualisé à vérifier } \\
\text { Actualisé par delà } \\
\text { Hors actualité } \\
\text { Pris comme actualité }\end{array}$ & $\begin{array}{l}\text { Supposé que } \\
\text { Dans la mesure où } \\
\text { En admettant que } \\
\text { A supposer que } \\
\text { En supposant que, des fois } \\
\text { que }\end{array}$ & $\begin{array}{l}\text { Du moment que } \\
\text { Pour autant que } \\
\text { Moyennant que } \\
\text { Sous réserve que } \\
\text { Pourvu que }\end{array}$ \\
\hline
\end{tabular}




\begin{tabular}{|l|l|l|l|}
\hline EVENTUALITES & Loterie & $P$ & Quand \\
& Si arrive, arrive & Au cas où & Dès lors que \\
& Pas nécessaire, mais & Que $\mathrm{p}$ & Pour peu que \\
possible & P ? & A condition que \\
& Possible parmi d'autres & Un coup que & Dès l'instant que \\
\hline
\end{tabular}

TABLEAU 2

\begin{tabular}{|c|c|c|}
\hline Vérifiable & Relationnel génér./ & Si un nombre est compris/ Si c'est un voilier... \\
\hline Vérifié & Métalinguistique & S'il est riche, il faut/ c'est parce... \\
\hline Avalisé & Thématique / Explicatif & S'il est riche, on peut/ il n'est pas... \\
\hline Mis en cause & & S'il était riche,... / S'il est riche, cela ne se \\
\hline Pris comme & Contrefactuel / Dialectique & voit pas. \\
\hline vérité & Fictif / Déductif & $\begin{array}{l}\text { Si je gagnais,... / S'il appelle, c'est qu'il a } \\
\text { quelque... }\end{array}$ \\
\hline
\end{tabular}

\section{NOTES}

1. A comparer avec le champ des circonstancielles temporelles où l'on distingue au moins assez clairement des marques de simultanéité, d'antériorité, de postériorité. Rien d'aussi net ici, excepté pour quelques marques de conditions négatives (comme à moins que).

2. A comparer avec le champ de la concession où il ne semble pas que l'une des locutions se distingue des autres comme étant plus prototypique.

3. de Vogüé, 1987a.

4. Les théories qui ont essayé de les réintégrer dans le champ de la condition ne peuvent le faire qu'en élargissant ce champ à tout énoncé circonstanciel : on explique alors que p désigne non pas une condition pour q mais une condition pour énoncer q, soit un cadre dans lequel le locuteur se place pour énoncer q ; une telle caractérisation vaudrait pour toute circonstancielle antéposée, l'antéposition marquant précisément que la circonstancielle figure le cadre de l'énoncé.

Sans doute pour montrer que (2-4) ne sont pas conditionnels faudrait-il disposer d'un critère fiable de ce qu'est une condition. Dans de Vogüé, à paraître, où j'examine le concept de condition, je propose un tel critère : la protase de (2-4) ne peut pas être glosée par la formule «dans ces conditions-là » sans que la valeur globale de l'énoncé n'en soit sensiblement déformée. Sur ce test, et sur le bien-fondé en général d'une démarche recourant au test des gloses, je renvoie à l'article en question.

5. Dans l'article cité note 2, je montre que les autres marqueurs restent, eux, dans le champ de la condition, y compris lorsqu'ilsexpriment une relation entre $\mathrm{p}$ et $\mathrm{q}$ du même ordre que celles quicaractérisent (2-4) (explication, opposition) :

A supposer qu'il soit riche, il n'est pas milliardaire.

s'avère être un énoncé conditionnel dont la protase peut être glosée par «dans ces conditionslà ".

6. Même dans les énoncés génériques, il est fréquent que l'énoncé ne concerne que les situations Sitp d'une période particulière. Qu'elles soient toutes parcourues n'empêche pas que chacune le 
soit dans sa particularité : on peut montrer que les énoncés génériques conditionnels ont nécessairement une interprétation distributive et non pas collective (de Vogüé, à paraître).

7. Sitp « valide » q et non pas « vérifie » q, puisqu'on a vu que q ne peut pas être tenu pour une propriété attribuée à Sitp (qui dès lors vérifierait q) : sur la différence entre vérification et validation, voir de Vogüé, 1995.

8. Il faut sans doute en fait distinguer une troisième valeur, mais qui ne semble pas jouer un rôle dans le champ des expressions conditionnelles : c'est la valeur que l'on trouve par exemple dans l'expression conditions de paiement, où il s'agit bien de la façon dont le paiement va se dérouler, mais où ces conditions sont stipulées plutôt qu'observées, et où il n'y aurait pas de sens à dire que le paiement est conditionné par la réalisation de ces conditions.

9. J'indique là que l'opposition que je cherche à mettre en évidence ne correspond pas malgré d'éventuelles apparences à l'opposition logique entre condition suffisante et condition nécessaire, dont j'ai montré par ailleurs qu'elle dépend beaucoup de facteurs extérieurs à l'expression même de la condition (en particulier la structure prédicative de l'énoncé), et qu'elle est en tout état de cause plus complexe en langue qu'elle ne l'est en logique (une condition peut être suffisante à différents titres, ou nécessaire de différentes façons (de Vogüé, 1987b). Cela n'empêche pas que parmi les marqueurs de conditions de validation, il y en ait qui vont dans le sens de la nécessité, et d'autres qui vont dans le sens de la suffisance

10. On voit que la condition est plutôt donnée comme nécessaire avec à condition que, mais comme simplement suffisante avec dès l'instant que. Avec pour peu que, la configuration est plus indécise : la locution marque à la fois que $\mathrm{p}$ (aussi « peu » soit-il) suffit à q, etque p est nécessaire (à cause du pour, qui peut s'interpréter comme indiquant qu'il faut $\mathrm{p}$, ou un équivalent, pour avoir q). On pourrait montrer qu'il n'y a pas deux valeurs - le suffisant et le nécessaire - mais plutôt tout un continuum, dont on ne peut rendre compte avec le seul moyen formel d'une orientation d'implication.

11. Il faut une configuration prosodique particulière pour que ce $p$ postposé fasse figure de prédicat (accent d'intensité sur si, sans pause entre q et p). Voir de Vogüé, 1987b.

12. Sur la différence entre discernement et singularisation (de Vogüé, 1993, ou Paillard et de Vogüé, à paraître), où cette différence est rapportée aux deux grands modes de constitution des occurrences d'une notion, ces deux modes étant donnés comme ce qui organise la théorie des domaines notionnels élaborée par l'école d'Antoine Culioli pour rendre compte de la complexité des opérations référentielles.

13. Vendler, 1957.

14. Ce nombre de cinq se rapporte au cinq valeurs que Denis Paillard et moi-même proposons d'attribuer à l'opération de repérage qui, dans le modèle culiolien, organise les opérations prédicatives et énonciatives (pour une présentation de ces cinq valeurs, voir en particulier de Vogüé, 1993). Il n'est pas possible dans le cadre de cet article d'élaborer cette théorie de repérage, dont on dira seulement ici les grandes lignes : la spécification de Sitp par p procède d'un repérage de Sitp par rapport à p ; ce repérage, qui identifie Sitp sur la classe des possibles, organise le rapport de Sitp à cette classe, et la structure dès lors diversement selon la façon dont l'identification de Sitp par $\mathrm{p}$ se trouve stabilisée ; il va par conséquent déterminer le statut de $\mathrm{p}$ par rapport aux possibles, c'est-à-dire, selon que les possibles sont des propositions ou des situations, son degré d'actualité ou son degré de possibilité. Qu'il y ait 5 plutôt que 4 ou 6 valeurs est une hypothèse empirique que nous formulons, et qui est fondée sur l'analyse du repérage comme procédant d'une identification sur fond d'altérité première : les 5 valeurs correspondent aux différentes façons de pondérer identité et altérité dans la stabilisation de cette identification. 15. On aura compris que, devant le nombre de tests et de descriptions intuitives à fournir pour autant de marqueurs et autant de critères, j'ai choisi de laisser au lecteur le soin de vérifier par lui-même mon classement, justifié simplement pour quelques marqueurs dans les considérations 
qui précèdent: normalement les mêmes tests, et les mêmes questions (est-ce plus ou moins possible ? est-ce plus moins actualisable ?) doivent être appliqués aux autres marqueurs.

16. Il faut donc qu'il y ait d'autres facteurs de différenciation, dont nous ne disons rien ici : on pourrait en particulier montrer que les marqueurs conditionnels se distinguent selon leur plus ou moins grande capacité à construire des énoncés génériques, ainsi que selon le caractère plus ou moins déterministe de la relation reliant $\mathrm{p}$ à $\mathrm{q}$ (ce qui se manifestera par exemple sous la forme de degrés divers de nécessité ou de suffisance).

17. de Vogüé, 1993.

18. Le classement que je propose ici des valeurs de si n'est pas le même que celui qui était présenté dans ma thèse et dans de Vogüé, 1987a. Les principes de différenciation aussi sont à la fois différents et plus nombreux : là où j'invoquais seulement l'instabilité propre à un repère fictif, je mobilise maintenant trois facteurs de déformation (condition initiale / condition de validation; éventualité / supposition ; énoncés relationnels / énoncés prédicatifs), et cinq relations possibles de la condition avec la classe dans laquelle elle s'inscrit (sans compter les points seulement évoqués ici concernant le degré de généricité de $\mathrm{p}$ et le caractère plus ou moins déterministe de la relation; sans compter aussi des paramètres extérieurs à la figure conditionnelle qui peuvent affecter l'interprétation finale). Cela tient au fait que j'ai été amenée à observer plus de distinctions, grâce à la confrontation de si avec les autres marqueurs du champ. Cela tient aussi au fait que le modèle dont je dispose maintenant est plus puissant, avec une théorie des repères qui s'est affinée et développée, et qui peut dès lors capter (modéliser) des différences plus fines. En particulier, c'est toute la catégorie que j'appelais «standard» qui s'avère recouvrir des types de valeurs extrêmement variés.

\section{RÉSUMÉS}

On montre que la configuration énonciative qui caractérise l'expression d'une relation conditionnelle est ambiguë, recouvrant deux types de condition d'une part, des éventualités et des suppositions d'autre part, des hypothèses et de simples prédications fictives enfin. Cela permet de rendre compte de la diversité des valeurs s'observant dans le champ des subordonnées conditionnelles du français, mais aussi de la relation de quasi synonymie qui pourtant unit ce champ, et de la position remarquable qu'y occupe la conjonction si, subsumant à elle seule toutes les différences rencontrées.

There is a fundamental ambiguity in the enunciative configuration that characterizes the expression of conditional relation: it is shown that this configuration covers two types of condition, an opposition between eventuality and supposition, an opposition between hypothesis and mere fictive predication. This triple ambiguity accounts for the diversity of values that can be encountered in French. It accounts also for the relation of quasi synonymy which unifies the field, as well as for the special position of the conjunction si in this field, when it alone can subsume all the distinctions observed. 
AUTEUR

SARAH DE VOGÜÉ

Université Paris X-Nanterre

URA 1028 CNRS

U.F.R. LLPhi

200 , avenue de la République

92000 - Nanterre Cedex 